\title{
Modelling and characterization of mechanical properties of optimized honeycomb structure
}

\author{
L. Zhang $\cdot$ B. Liu $\cdot$ Y. Gu $\cdot$ X. H. Xu
}

Received: 2 November 2018/ Accepted: 13 May 2019

(C) Springer Nature B.V. 2019

\begin{abstract}
The honeycomb structure can be applied to the design of new lightweight composites materials due to its excellent properties such as high strength, strong energy absorption ability, low thermal conductivity, etc. In recent years, researchers have noticed that cell joint performance optimization can improve the performance of honeycomb structures. In this study, an optimized honeycomb structure with cell joint thickened was obtained by a theoretical analysis in which two principal geometrical parameters were adjusted, and then fabricated with additive manufacturing technology for compression testing. The results were obtained through experimentation and qualitative simulation, and then compared with those of a traditional honeycomb structure with uniform wall thickness. Finally, the mechanism of the optimized thickened-joint honeycomb structure was investigated, which could be a design guideline for the application of honeycomb structures in various engineering fields.
\end{abstract}

Keywords Honeycomb structure - Cell joint thickened $\cdot$ Mechanical properties optimization

L. Zhang · B. Liu · Y. Gu · X. H. Xu ( $)$

Institute of Mechanics, Chinese Academy of Sciences, No. 15, North Sihuan West Road, Beijing 100190, China e-mail: xxh@1nm.imech.ac.cn

\section{Introduction}

As one of the most commonly used cores for sandwichstructured materials, the honeycomb structure and derived cell-shaped structure have been widely used for developing lightweight yet high-strength structures in many industries, such as architecture, transportation, mechanical engineering, chemical engineering, nanofabrication and, more recently, biomedicine (Zhang and $\mathrm{Li}$ 2018; Uchiyama and Nakashima 2013; Huang et al. 1997; Zeng et al. 2018; Jin et al. 2018). Honeycomb structures exhibit distinctive and excellent properties, such as light weight, high strength and strong energy absorption ability, and thus have received much attention (Huang et al. 1999; Wen et al. 2017; Abd Kadir et al. 2017). For example, Gibson and Ashby explored the relationship between relative density, cell wall thickness and geometrical shape of unit cells, and proved that the mechanical properties of honeycomb structures were dominated by these aforementioned parameters (Gibson and Ashby 2014). Hu et al. (2013) studied the effect of cell wall angle on the compressive properties of honeycomb structures, through experimentation and numerical simulations. Jin et al. (2016) investigated the effects of size on the in-plane mechanical properties of aluminum hexagonal honeycombs. Hedayati et al. (2016) studied the mechanical properties of honeycomb structures with different wall thickness. Galehdari et al. (2015) reported their results of research on a graded honeycomb structure under low-velocity impact and quasi-static in-plane loading conditions. All these studies 
mentioned above and even most studies under progress concentrate on traditional hexagonal honeycomb structures with uniform cell wall thickness. In recent years, researchers have come to realize the important of cell joints of the honeycomb structure because it is the joints that bear the largest bending moment under loading condition. For this reason, various optimized designs of the cell joint were developed to improve the mechanical properties of honeycomb structures. For example, Chen et al. (2014) constructed a hollow-cylindrical joint in honeycomb structures, and Ajdari et al. (2012) investigated the hierarchical-structure design of cell joints in honeycomb structure.

Thickened-joint honeycomb structure is discussed in this manuscript. Theoretical analysis, numerical simulation and experiment results show that cell joint thickening can effectively improve the compressive performance of honeycomb structures. This might be guidelines for applications of honeycomb structure in engineering fields.

\section{Theoretical relation in equivalent elastic modulus}

Since there are higher stress at the end part of the honeycomb cell wall and lower stress at the middle part (Gibson and Ashby 2014), theoretically, the thickened-joint hexagonal unit cell may have better mechanical properties than those of the uniformthickness cell wall. For thickened-joint cell walls, each wall can be regarded as an individual non-prismatic beam. As shown in Fig. 1a, the wall length and the angle between the $x$ axis and the diagonal bar are denoted as $l$ and $\theta=30^{\circ}$, respectively. In the thickened-joint cell wall, the ratio of the middle part length to the end part length can be denoted as $\xi(\xi \geq 0)$ and therefore the sum of the lengths of the middle and end parts can be given by $(1+2 / \xi) l_{H}=l$, where $l_{H}$, and $l_{H} / \xi$ represent the lengths of the middle and end parts of the cell wall, respectively. Similarly, the ratio of the middle part thickness to the end part thickness can be denoted as $\eta(0<\eta \leq 1)$, hence the thicknesses of the middle and end parts can be expressed as $t_{H}$ and $t_{H} / \eta$, respectively. Next, the second moment of inertia of the middle and end sections on the $z$ axis in the wall, can be given as $I=b t_{H}^{3} / 12$ and $I_{d}=1 / \eta^{3} I$, respectively, assuming that the cell wall has a rectangular crosssection with depth $b$.
As shown in Fig. 1b, assuming that the compressive stress in the thickened-joint honeycomb structure system in the $y$ axial direction is uniaxial, the force condition at the two endpoints $\mathrm{A}$ and $\mathrm{B}$ of the simplified inclined wall can be represented as

$W=\sigma b l \cos \theta$

$M=\frac{W l \cos \theta}{2}$

where $\sigma$ indicates the uniform compressive stress applied in the $y$ axial direction, while $W$ and $M$ represent $y$ axis direction force and bending at Point A or Point B, respectively.

Based on the Euler-Bernoulli's beam theory, the strain energy in the inclined bar can be expressed as (Gibson and Ashby 2014)

$$
\begin{aligned}
U= & \int_{0}^{l} \frac{M(x)^{2}}{E_{\mathrm{S}} I} \mathrm{~d} x \\
= & \int_{0}^{l_{\mathrm{H}} / \xi} \frac{(M-W \cos \theta \cdot x)^{2}}{E_{\mathrm{S}} I_{d}} \mathrm{~d} x \\
& +\int_{l_{\mathrm{H}} / \xi}^{l_{\mathrm{H}} / \xi+l_{\mathrm{H}}} \frac{(M-W \cos \theta \cdot x)^{2}}{E_{\mathrm{S}} I} \mathrm{~d} x \\
& +\int_{l_{\mathrm{H}} / \xi+l_{\mathrm{H}}}^{l} \frac{(M-W \cos \theta \cdot x)^{2}}{E_{\mathrm{S}} I_{d}} \mathrm{~d} x
\end{aligned}
$$

where $E_{s}$ indicates the elastic modulus of the solid material.

Substituting Eqs. (1) and (2) into the energy Eq. (3) and then using the Castigliano's theorem, we obtain the vertical displacement at Point $\mathrm{A}$ in the diagonal wall:

$\delta=\frac{\partial U}{\partial W}=\frac{W l^{3} \cos \theta}{E_{\mathrm{S}} I} \frac{\xi^{3}+8 \eta^{3}+12 \xi \eta^{3}+6 \xi^{2} \eta^{3}}{12(2+\xi)^{3}}$

By fixing Point $\mathrm{B}$ and ignoring the deformation of walls in the axial and tangential directions, the strain in the unit cell in the axial direction can be expressed as 
Fig. 1 Diagram of thickened-joint honeycomb structure: a unit cell is subjected to axial stress $\sigma$ in the $y$ direction; $\mathbf{b}$ the simplified force of the diagonal wall

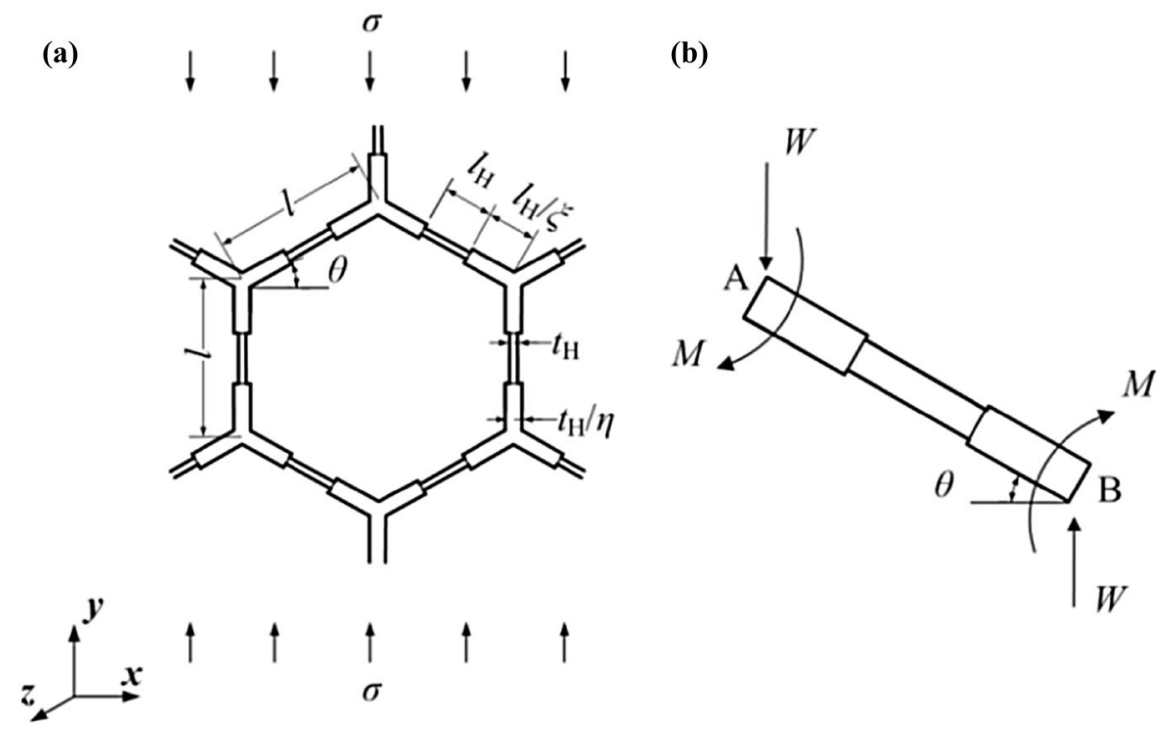

$$
\begin{aligned}
\varepsilon & =\frac{\delta \cos \theta}{l+l \sin \theta} \\
& =\frac{\sigma b l^{3} \cos ^{3} \theta}{E_{\mathrm{s}} I(1+\sin \theta)} \frac{\xi^{3}+8 \eta^{3}+12 \xi \eta^{3}+6 \xi^{2} \eta^{3}}{12(2+\xi)^{3}}
\end{aligned}
$$

from which the equivalent elastic modulus of the unit cell parallel to the $y$ direction can be simply written as

$E_{\mathrm{H}}=\frac{\sigma}{\varepsilon}$

Then substituting Eqs. (5) into the equivalent elastic modulus Eq. (6) yields

$\frac{E_{\mathrm{H}}}{E_{\mathrm{s}}}=\left(\frac{t_{\mathrm{H}}}{l}\right)^{3} \frac{(1+\sin \theta)(2+\xi)^{3}}{\cos ^{3} \theta\left(\xi^{3}+8 \eta^{3}+12 \xi \eta^{3}+6 \xi^{2} \eta^{3}\right)}$

From the Eq. (7) above, an interesting rule can be found that the value of the equivalent elastic modulus of the unit cell depends on the length $l, t_{H}$ and the ratios $\xi$ as well as $\eta$ in the periodic unit cell.

In the present study, one specific triangular region around the common joint of adjacent cells is selected, as shown in Fig. 2. The area of it can be expressed as $A_{h}=\sqrt{3} l \times 3 l / 2 \times 1 / 2=3 \sqrt{3} l^{2} / 4$. Identically, the area of bars in the thickened-joint honeycomb structure system can be expressed as $A_{b}=3\left(l_{H} t_{H} / \xi \eta+\right.$ $\left.l_{H} t_{H} / 2\right)$. Hence, the relative density of the thickenedjoint honeycomb structure $\rho_{H} / \rho_{S}$ can be written as

$\frac{\rho_{\mathrm{H}}}{\rho_{\mathrm{s}}}=\frac{A_{b}}{A_{h}}=\frac{3\left(\frac{l_{\mathrm{H}} t_{\mathrm{H}}}{\xi \eta}+\frac{l_{\mathrm{H}} t_{\mathrm{H}}}{2}\right)}{3 \sqrt{3} l^{2} / 4}$

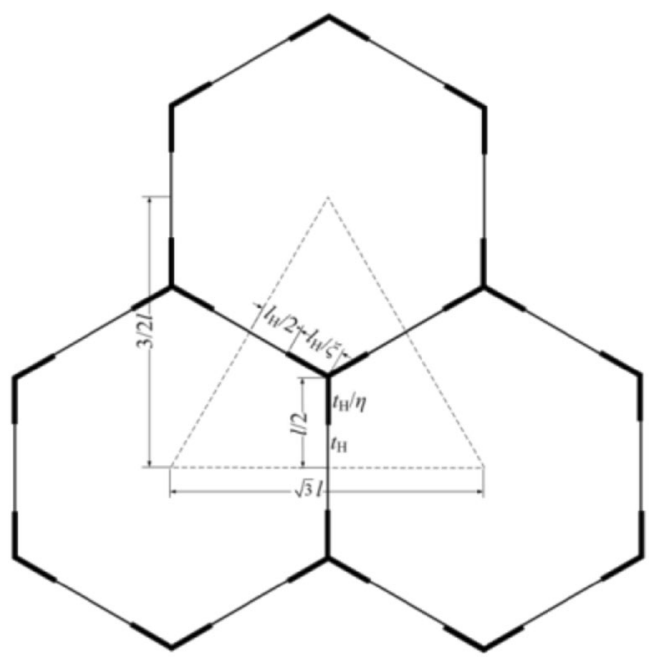

Fig. 2 The schematic diagram of the relative density calculation

where $\rho_{H}$ indicates the equivalent density of the thickened-joint honeycomb structure, and $\rho_{s}$ indicates the density of the solid material.

Based on the theory of a uniform thickness hexagonal cell wall by Gibson and Ashby (2014), when $\rho / \rho_{s}<0.3$, the relative density of the uniform thickness honeycomb structure $\rho / \rho_{s}$ can be expressed as

$\frac{\rho}{\rho_{s}}=\frac{2}{\sqrt{3}} \frac{t}{l}$ 
where $\rho$ indicates the effective density of the regular honeycomb structure.

In this study, it is assumed that the relative density of the thickened-joint hexagon is the same as that of the regular hexagon, which means the same amount of material is used, thus

$\frac{\rho_{H}}{\rho_{S}}=\frac{\rho}{\rho_{S}}$

From the above, the expression of $t_{H}$, the thickness value of the middle region in the thickened joint honeycomb wall, can be written as

$t_{H}=t\left(\frac{2 \eta+\xi \eta}{2+\xi \eta}\right)$

Moreover, based on the investigation by Gibson and Ashby, the relationship between the equivalent elastic modulus $E$ of the regular cell structure and the elastic modulus $E_{s}$ of the solid material can be expressed as

$\frac{E}{E_{s}}=\frac{4}{\sqrt{3}}\left(\frac{t}{l}\right)^{3}$

Combining Eqs. (7), (11) and (12), we can obtain the specific ratio of the thickened-joint hexagon elastic modulus and the regular hexagon elastic modulus:

$\frac{E_{H}}{E}=\frac{(2+\xi)^{6} \eta^{3}}{(2+\xi \eta)^{3}\left(\xi^{3}+8 \eta^{3}+12 \xi \eta^{3}+6 \xi^{2} \eta^{3}\right)}$

From Eq. (13), it is found that the value of elastic modulus ratio $E_{H} / E$ merely correlates to the parameters $\xi$ and $\eta$. Figure 3 shows the relationship of the elastic modulus ratio $E_{H} / E$ to the variables of $\xi$ and $\eta$.

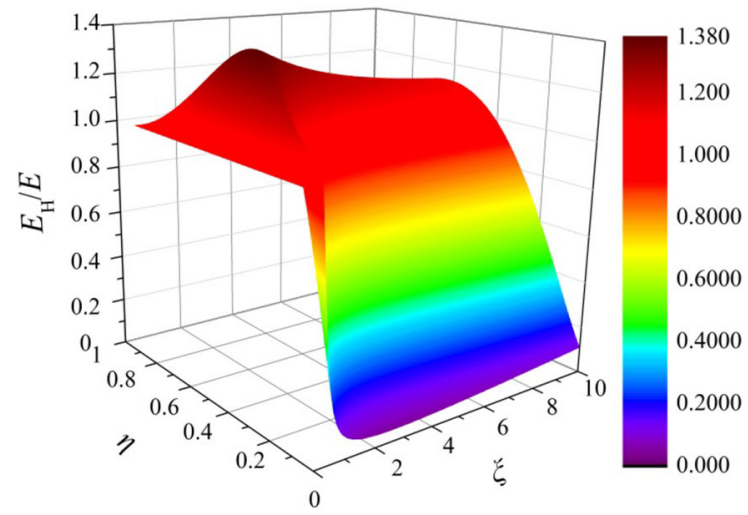

Fig. 3 The elastic modulus ratio $E_{H} / E$ in terms of the variables of $\xi$ and $\eta$
From Eq. (13), when $\xi=1.125$ and $\eta=0.545$, the ratio reaches the maximum value $E_{H} / E=1.378$. Besides, under the conditions $\xi=1.125$ and $0.326<\eta \leq 1$, the elastic modulus ratio $E_{H} / E>1$, as shown in Fig. 4a. From Fig. $4 b$, it is found that when $\eta=0.545$ and $\xi>0$, the elastic modulus ratio $E_{H} / E>1$.

Based on the analysis above, the equivalent elastic modulus of optimized thickened-joint hexagon can be enhanced by adjusting the geometrical parameters $\xi$ and $\eta$, and the maximum value can reach 1.378.

\section{Experiment}

\subsection{Sample processing and testing}

The thickened-joint honeycomb structure samples tested in the present study are fabricated by Lian Tai RS Pro600 SLA 3D printer (printing accuracy: $0.02-0.1 \mathrm{~mm}$ ) and the print material is Somos EvoLVe 128 (Farahani et al. 2014; Stansbury and Idacavage 2016). Figure 5 displays the diagram of a printed sample consisting of $9 \times 6$ thickened-joint hexagons set at the relative density condition $\rho_{H} / \rho_{s}=10.58 \%<30 \%$. The width of the system is $L_{1}=145.49 \mathrm{~mm}$, the height $L_{2}=196.00 \mathrm{~mm}$, the thickness $L_{3}=40.00 \mathrm{~mm}$ and the length of cell wall $l=14.00 \mathrm{~mm}$. In order to alleviate the concentration of stress caused by the sudden change in thickness, the variation in thickness of the cell bar is designed in a trapezoid manner. Six groups of samples (one group with uniform thickness walls and other groups with different thicknesses walls) are fabricated and their parameters are shown in Fig. 6 and Table 1.

In this study, a MTS Landmark testing machine, with maximum load of $50 \mathrm{kN}$ and loading rate of $6 \mathrm{~mm} / \mathrm{min}$, was used to measure the mechanical properties of thickened-joint honeycomb test samples in the quasi-static uniaxial compression experiment (Li et al. 2015, 2017; Forquin et al. 2000). In order to reduce the influence of friction, which can significantly affect the stress on the contact surfaces between the sample and platen, lubricating oil was smeared on the contact surfaces. During the loading procedure, as shown in Fig. 7, a three-dimensional high-resolution strain optical measurement system (DOM 3D LTD) sampling frequency of $1 \mathrm{fps}$ was utilized to capture the deformed configurations of the specific interest. 

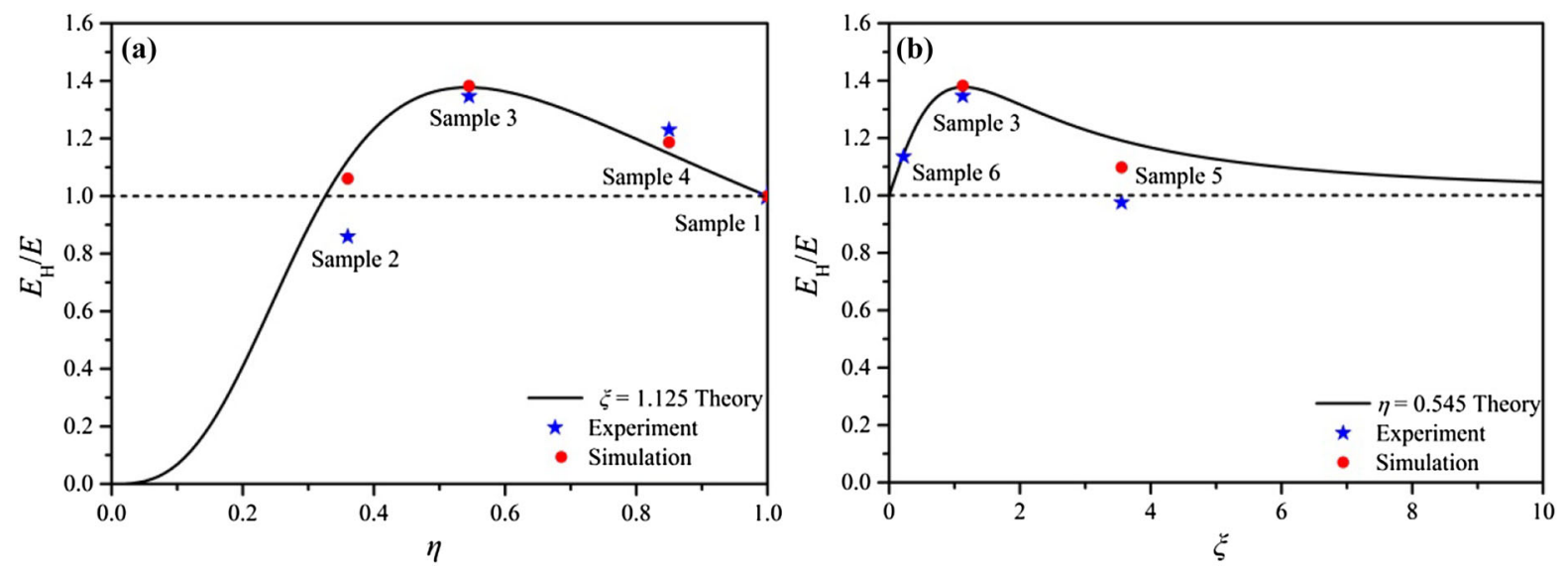

Fig. 4 The curves of elastic modulus ratio for $\mathbf{a} \xi=1.125$ and $\mathbf{b} \eta=0.545$

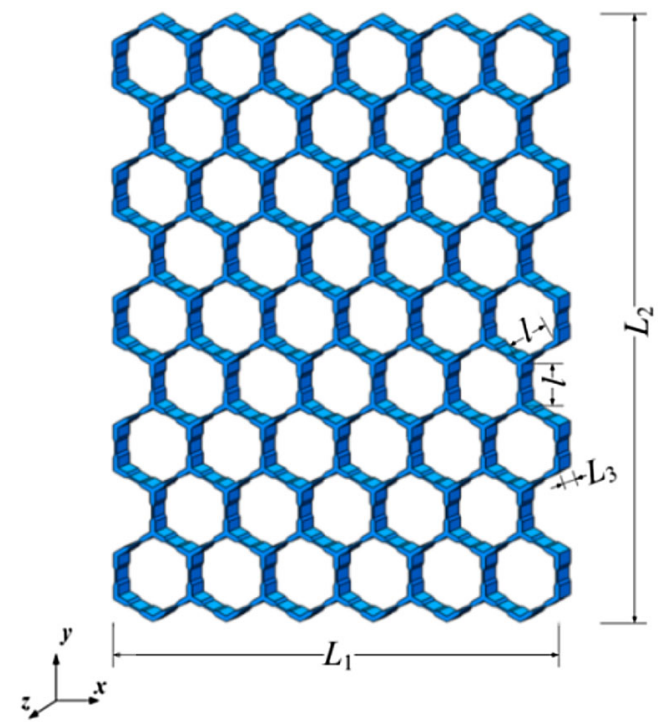

Fig. 5 Schematic diagram of printed thickened-joint hexagon system

\subsection{Experiment results and discussion}

As shown in Fig. 4, the mark $\star$ represents the distribution of the elastic modulus ratio of the thickened-joint honeycomb structures. It's observed that, the elastic modulus ratios of Sample 3, Sample 4 and Sample 6 were basically consistent with the theoretical values. Nevertheless, there were significant differences between the experimental and theoretical values of the elastic modulus ratios of Sample 2 $(\xi=1.125, \eta=0.360)$ and Sample $5(\xi=3.556$, $\eta=0.545$ ), due to the significant difference in
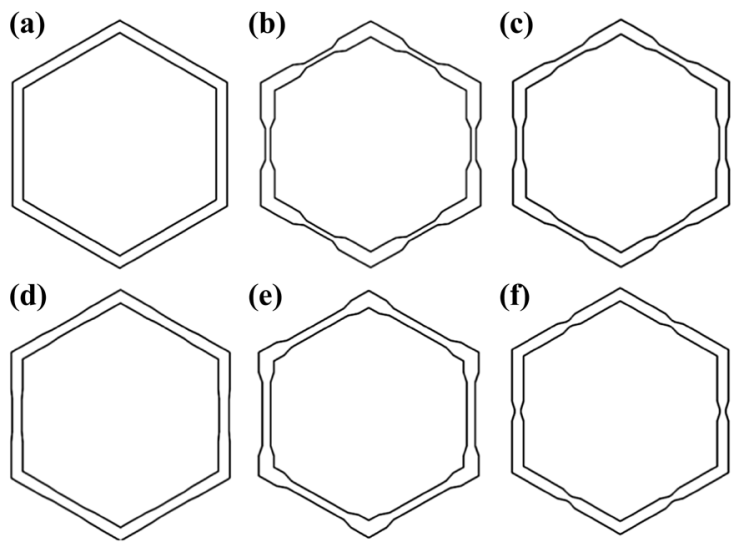

Fig. 6 The hexagonal unit cells. a the uniform thickness; b $\xi=1.125, \eta=0.360 ; \mathbf{c} \xi=1.125, \eta=0.545 ; \mathbf{d} \xi=1.125, \eta=$ $0.850 ; \mathbf{e} \xi=3.556, \eta=0.545 ; \mathbf{f} \xi=0.222, \eta=0.545$

thickness ratio and thickness mutation between the middle and end parts in the honeycomb walls.

With a simple treatment applied to the recorded load-displacement curves, the experimental stressstrain curves $\left(\sigma=P / L_{1} L_{3}\right.$, where $P$ is the vertical load; $\varepsilon=\delta / L_{2}$, where $\delta$ is the vertical displacement) of the regular hexagon and the thickened-joint honeycomb structures were obtained, as shown in Fig. 8a. From the above, the equivalent elastic modulus of each specimen can be calculated. The equivalent elastic modulus of the uniform-thickness honeycomb structure (Sample 1) was $2.19 \mathrm{MPa}$ and its peak stress was $0.20 \mathrm{MPa}$. As expected, compared to the uniform-thickness honeycomb structure, the thickened-joint honeycomb structures had better mechanical properties, such as higher peak stress 
Table 1 Cellular elements with different size parameters

\begin{tabular}{lllllll}
\hline Sample no. & $\xi$ & $\eta$ & $t_{H}(\mathrm{~mm})$ & $t_{H} / \eta(\mathrm{mm})$ & $l_{H}$ & $l_{H} / \xi$ \\
\hline 1 & - & 1.000 & 1.283 & - & - & - \\
2 & 1.125 & 0.360 & 0.600 & 1.667 & 5.040 & 4.480 \\
3 & 1.125 & 0.545 & 0.836 & 1.534 & 5.040 & 4.480 \\
4 & 1.125 & 0.850 & 1.153 & 1.356 & 5.040 & 4.480 \\
5 & 3.556 & 0.545 & 0.986 & 1.810 & 8.960 & 2.520 \\
6 & 0.222 & 0.545 & 0.732 & 1.344 & 1.399 & 6.301 \\
\hline
\end{tabular}

Fig. 7 a Experimental diagram and b principal experimental setup for compression test of thickened-joint honeycomb structure
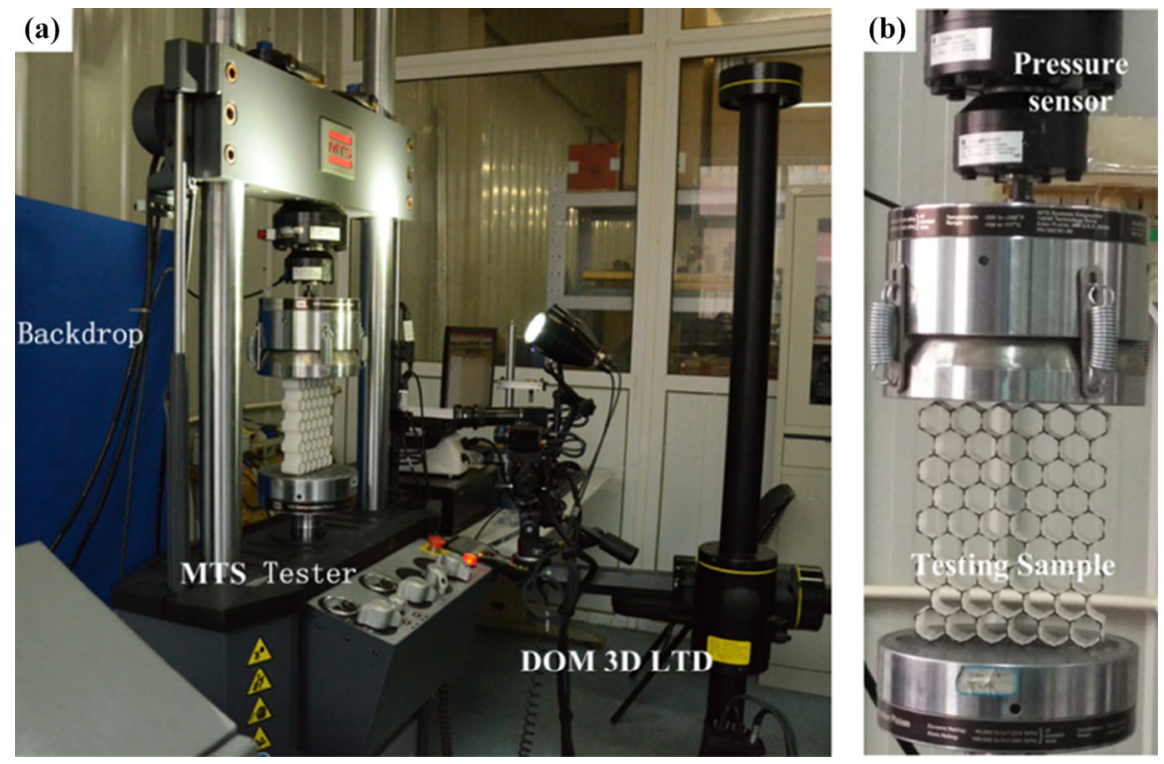

and greater elastic modulus. Particularly, Sample 3 $(\xi=1.125, \eta=0.545)$ had the maximal equivalent elastic modulus among all the testing samples, about $30 \%$ higher than that of the uniform-thickness honeycomb structure. Meanwhile, the equivalent elastic modulus and the peak stress of the testing sample 4 $(\xi=1.125, \eta=0.850)$ are $22 \%$ and $9.35 \%$ higher than those of the uniform-thickness honeycomb structure, respectively. Thus, it can be concluded that thickening joint can effectively improve the equivalent elastic modulus and the peak stress of the honeycomb structure, and that honeycomb structures with thickened joint have the same stability under the same relative density condition.

The stress-strain curves in Fig. 8a and deformation process of the uniform thickness honeycomb structure (Sample1) in Fig. 8b are exactly similar to the results provided by Papka and Kyriakides (1994) (aluminum honeycomb structure) and Bates (thermoplastic polyurethane honeycomb structure) Bates et al. (2016ab, ).
From the recorded deformation processes of the testing samples, a very interesting phenomenon can be observed that each area of the joint with the three connected bars is like a ' $\mathrm{Y}$ ' shaped unit cell and the local deformation of the ' $\mathrm{Y}$ ' domain rotates around the joint, while the wall of the hexagonal cell lost its bearing capacity until the structure of cell became more compact (Chen et al. 2018). Due to the buckling failure that occurred in the joint, corresponding with the local collapse, the deformation spreads to the two neighboring rows of cells while other rows remain unaffected. With the load increasing, further deformation is required in the deformed rows to make the walls of the collapsed cells contact each other, which allows the deformation to extend on both sides. In addition, all compression modes of the testing samples had three typical stages: (1) linear-elastic stage, in which all unit cells have a homogeneous and symmetric deformation; (2) stress-platform area, in which after reaching the maximum stress, local deformation 

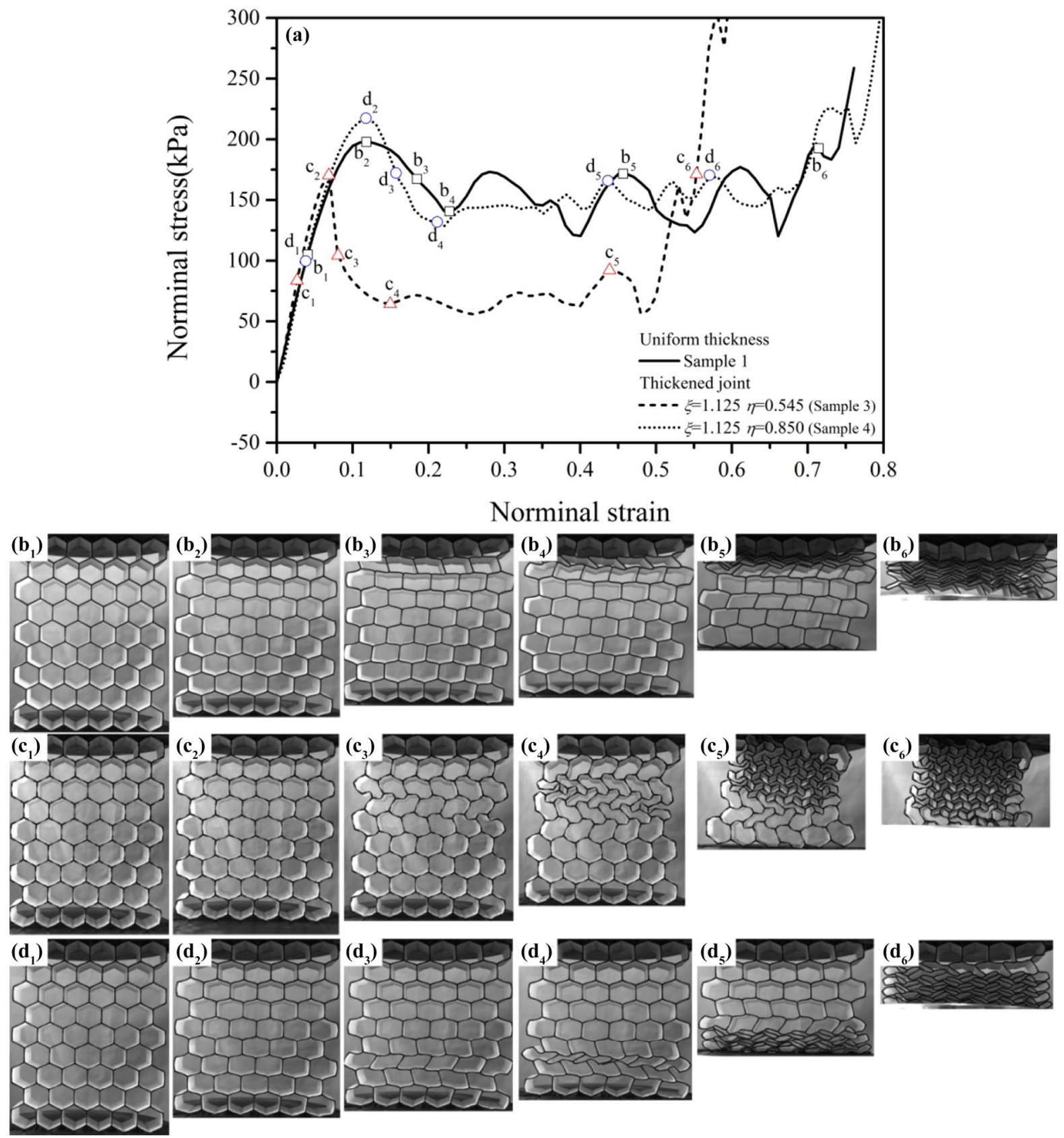

Fig. 8 a Quasi static compression experimental stress-strain curves; $\mathbf{b}_{1}-\mathbf{b}_{6}$ deformation process of uniform-thickness honeycomb structure Sample $1 ; \mathbf{c}_{1}-\mathbf{c}_{6}$ deformation process of

thickened-joint honeycomb structure Sample $3(\xi=1.125$, $\eta=0.545) ; \quad \mathbf{d}_{1}-\mathbf{d}_{6}$ deformation process of thickened-joint honeycomb structure Sample $4(\xi=1.125, \eta=0.850)$

of the samples occurs, and with the load increasing, some rows of the hexagonal cells system begin to collapse asymmetrically; and (3) close-grained stage, in which since further deformation is required with the strain increasing, the walls of the collapsed cells come

into contact, which causes the stress value to rise rapidly and correspond to the systematic densification (Wang and Yao 2013; Li et al. 2018; Kobayashi et al. 2011). 
Remarkably, in Fig. 8a a platform occurs in the stress-strain curve of the thickened-joint sample 3 $(\xi=1.125, \eta=0.545)$, although the peak stress and the platform are both lower than those of the uniformthickness honeycomb structure. However, for the thickened-joint Sample 4 ( $\xi=1.125, \eta=0.850)$, the platform of the stress-strain curve is approximately the same high as that of Sample 1, while the peak stress is higher than that of Sample 1. The reason is that the undersized ratio of the middle part thickness to the end part thickness (exaggerated thickness difference) causes the end part to experience strong constraint and small deformation. Meanwhile, the thin middle part in the cell wall can easily lose stability and generate a large amount of deformation, as shown in Fig. 9.

\section{Numerical simulation and results}

\subsection{Computational model}

In the present work, ABAQUS, the finite element software, was used to investigate quasi-static uniaxial compression behavior in established honeycomb
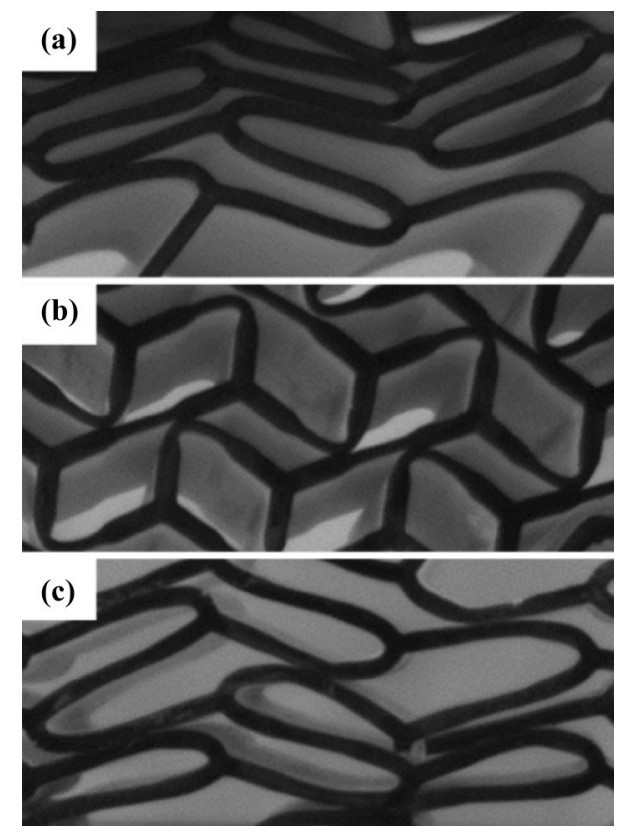

Fig. 9 Collapse configuration of honeycomb cells; a uniform thickness honeycomb Sample 1, b thickened-joint honeycomb sample $3(\xi=1.125, \eta=0.545)$, $\mathbf{c}$ thickened-joint honeycomb sample $4(\xi=1.125, \eta=0.850)$

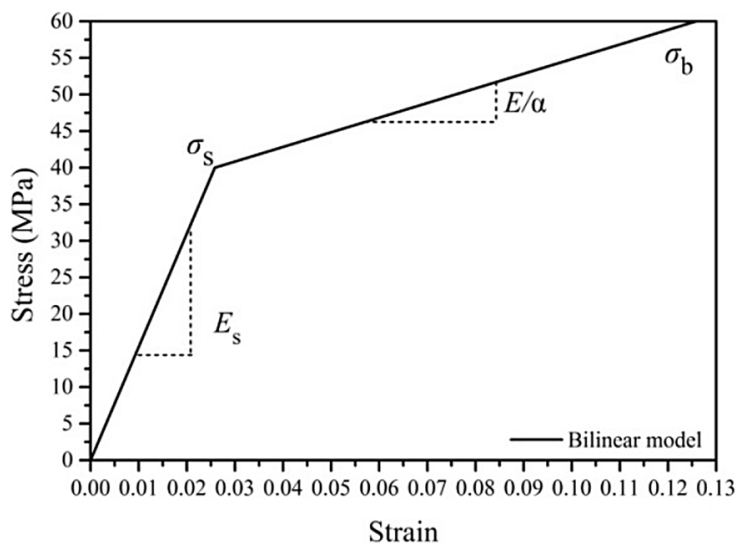

Fig. 10 Constitutive model of material in honeycomb structure simulation

structures in the $y$ direction (Alderson et al. 2010; Ali et al. 2008; Ippili et al. 2003). Figure 5 demonstrates that the geometrical parameters of a computational model which is the same as the experimental sample. Moreover, the bilinear elastoplastic constitutive model (Car et al. 2000; Cao and Li 2017; Zhang and Ruan 2012), as shown in Fig. 10, was used to model the solid material (Somos EvoLVe 128). It can be seen in Fig. 10 that the stress-strain relationship accords with the linear elasticity when the stress is lower than the yield limit. When the stress is higher than the yield limit, a linear strain-hardening stage is then assumed. In Table 2, the geometrical parameters and physical parameters such as the elasticity modulus, yield stress, subsequent yield modulus and

Table 2 Basic parameters of the numerical model

\begin{tabular}{ll}
\hline Geometrical size & Value \\
\hline Relative density $(\%)$ & 10.58 \\
Cell number & $9 \times 6$ \\
Width of model $L_{1}(\mathrm{~mm})$ & 145.49 \\
Height of model $L_{2}(\mathrm{~mm})$ & 196.00 \\
Thickness of model $L_{3}(\mathrm{~mm})$ & 40.00 \\
Length of cell wall $l(\mathrm{~mm})$ & 14.00 \\
Constitutive model & \\
Elastic modulus $E_{s}(\mathrm{MPa})$ & 1550 \\
Yield stress $\sigma_{s}(\mathrm{MPa})$ & 40 \\
Yield elastic modulus $E / \alpha(\mathrm{MPa})$ & 200 \\
Tensile strength $\sigma_{b}(\mathrm{MPa})$ & 60 \\
\hline
\end{tabular}


strength of extension in the honeycomb structure is tabulated for the finite element simulation. The parameters for simulation were selected from the experimental results and the data provided by the $3 \mathrm{D}$ printing company.

With these geometrical parameters and material properties above, the three-dimensional finite element simulation was performed in ABAQUS. To realize the compression of the honeycomb structure, two rigid platforms were introduced to represent the above and bottom planes, as shown in Fig. 11. In the simulation, the bottom rigid plane was fixed and the upper rigid plane moved down to simulate the compression process.

\subsection{Results and discussion}

The values of elastic modulus ratios $E_{H} / E$ of thickened-joint honeycomb obtained by numerical simulation, marked as in Fig. 4, were very close to the theoretical values and the experimental results. To demonstrate the uniaxial compression procedure of the honeycomb structure, the uniform thickness Sample 1 and the thickened-joint Sample $4(\xi=1.125$, $\eta=0.850)$ are taken as examples. The corresponding stress-strain curves as well as the deformation history are shown in Fig. 12, from which it can be seen that the appropriate thickened-joint design not only enhances the equivalent elastic modulus of the honeycomb structure, but also effectively increases its peak stress. Likewise, there are three stages in the computational compression procedure of the uniform thickness and

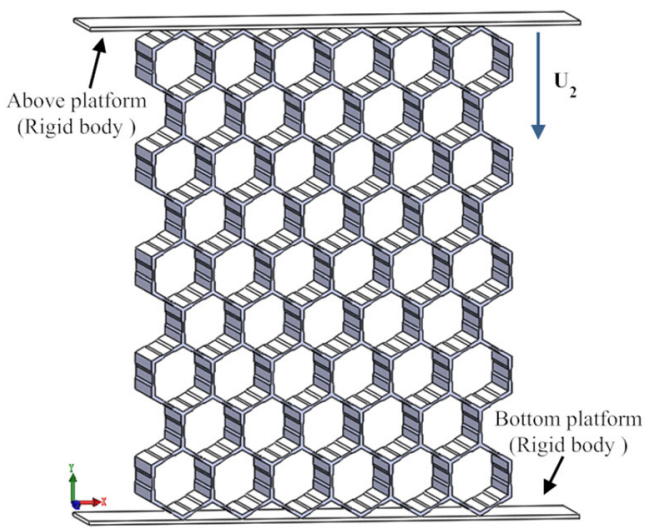

Fig. 11 Finite element model for thickened-joint honeycomb structure with rigid upper and lower boundaries
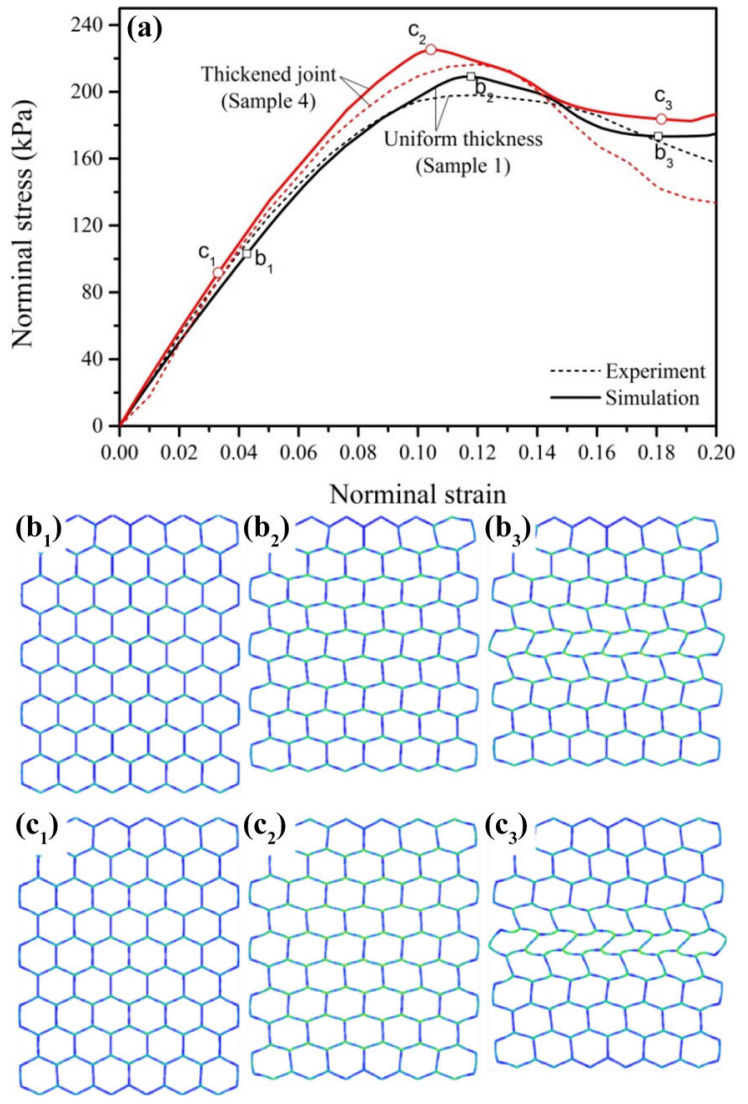

Fig. 12 a Stress-strain curves; $\mathbf{b}_{1}-\mathbf{b}_{3}$ deformed configurations of uniform thickness honeycombs in compression simulation; and $\mathbf{c}_{1}-\mathbf{c}_{3}$ deformed configurations of thickened-joint honeycombs in compression simulation

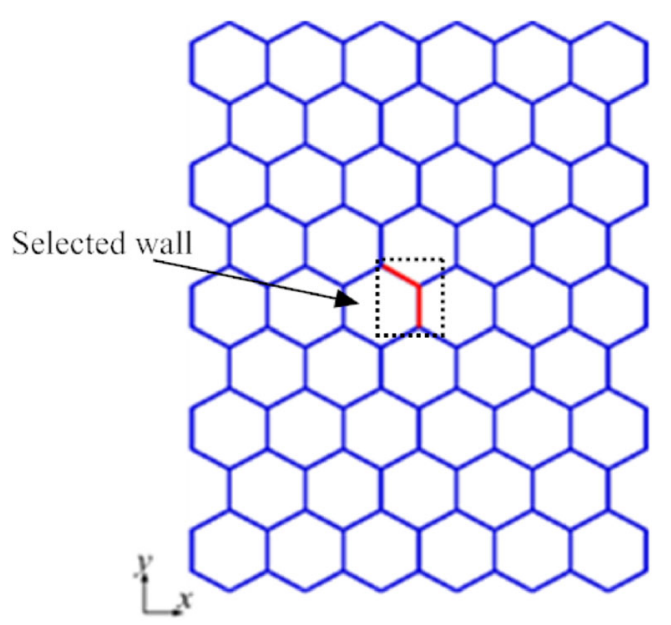

Fig. 13 The position of the selected walls in the honeycomb structure 
thickened-joint honeycomb structures: (1) homogeneous deformation, (2) local failure, (3) dense phase in walls.

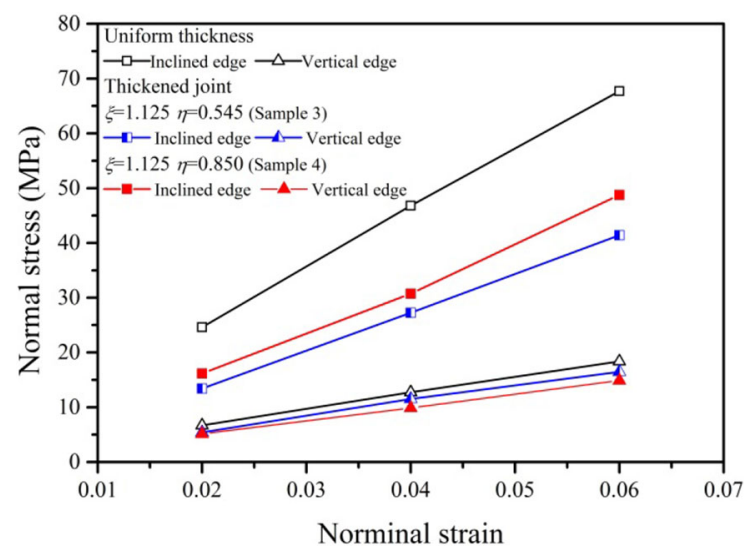

Fig. 14 The variation trend of stress difference in the cell wall under incremental nominal strain
Additionally, in order to investigate the reason why the thickened-joint design can improve the equivalent elastic modulus and platform stress of the honeycomb structure system, one specific diagonal edge and one vertical edge connected in the middle cell joint were selected for further study (see red lines in Fig. 13). With the increase of nominal strain, the maximum and minimum stress values in the diagonal edge and the vertical edge are calculated and plotted in Fig. 14 (Hadjistamov 1995; Yang and Ravi-Chandar 1999). It can be seen that the stress difference of the diagonal wall and the vertical wall becomes bigger with the increase of nominal strain for the three computational models and the stress value of the diagonal wall is always greater than that of the vertical wall. Moreover, it should be noted that the stress difference of the diagonal edge in the thickened-joint honeycomb is always smaller than that in the uniform-thickness honeycomb structure. Thus, it can be concluded that
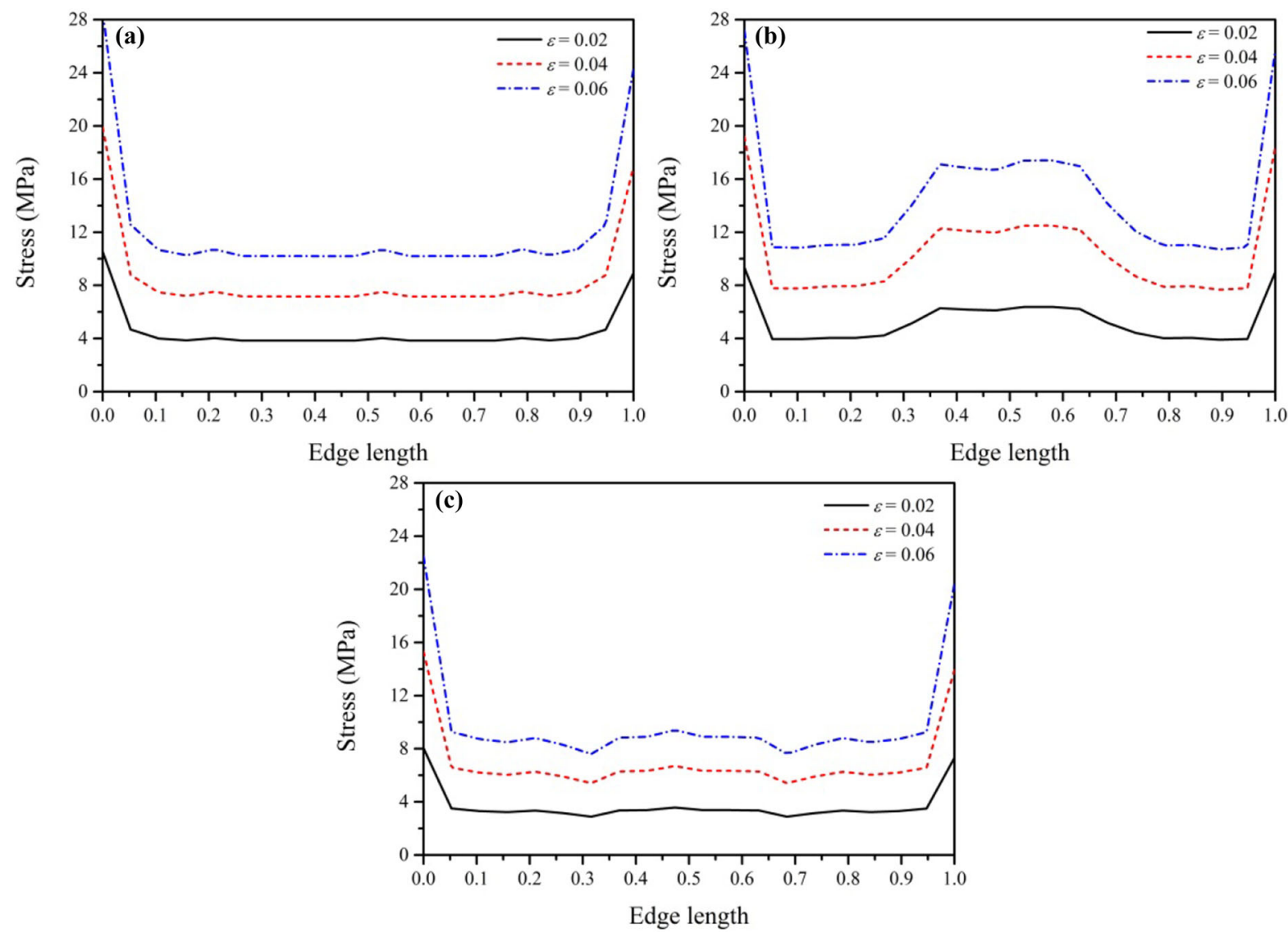

Fig. 15 The stress distribution in the vertical edge under different nominal strain: a uniform thickness honeycomb (Sample 1); b thickened-joint honeycomb Sample $3(\xi=1.125, \eta=0.545)$; c thickened-joint honeycomb Sample $4(\xi=1.125, \eta=0.850)$ 
the thickened-joint design can improve the mechanical properties and stability of honeycomb structures, as compared with the uniform-thickness honeycomb structure.

Furthermore, the stress distributions in the vertical wall of the uniform-thickness and thickened-joint honeycombs are depicted in Fig. 15, in which the length of vertical wall is non-dimensional and falls in the range of $[0,1]$ (Kim et al. 2017). It is founds that the stress rises with the increase of nominal strain in honeycomb structures. Interestingly, the value of stress in the thickened-joint structure Sample 4 ( $\xi=1.125, \eta=0.850)$ is lower than that in Sample $3(\xi=1.125, \eta=0.545)$ and the uniform-thickness structure Sample 1. This is the main reason why Sample 4 has better stability than Sample 3.

\section{Conclusions}

In this paper, a thickened-joint honeycomb structure is introduced. By combining theory, calculation and experiment, the deformation process and strengthening mechanism are studied. The results showed that the thickened-joint design can optimize the stress distribution of cell wall as the structure is subjected to external forces, and effectively improve the compressive strength and stability of honeycomb structures. And based on the improved performance, more farreaching influence and structural application of honeycomb structure can be expected in various industries.

Funding Funding was provided by National Natural Science Foundation of China (CN) (No. 11672297) and Strategic Priority Research Program of the Chinese Academy of Sciences (No. XDB22020200).

\section{References}

Abd Kadir, N., et al.: Experimental study of low-velocity impact on foam-filled Kraft paper honeycomb structure. In: International Conference on Advances in Manufacturing and Materials Engineering 2017, ICAMME 2017, August 8, 2017-August 9, 2017. Institute of Physics Publishing, Kuala Lumpur (2018)

Ajdari, A., et al.: Hierarchical honeycombs with tailorable properties. Int. J. Solids Struct. 49(11-12), 1413-1419 (2012)

Alderson, A., et al.: Elastic constants of 3-, 4- and 6-connected chiral and anti-chiral honeycombs subject to uniaxial in- plane loading. Compos. Sci. Technol. 70(7), 1042-1048 (2010)

Ali, M., et al.: Theoretical and finite element study of a compact energy absorber. Adv. Eng. Softw. 39(2), 95-106 (2008)

Bates, S.R.G., Farrow, I.R., Trask, R.S.: 3D printed elastic honeycombs with graded density for tailorable energy absorption. In: Active and Passive Smart Structures and Integrated Systems 2016, March 21, 2016-March 24, 2016. SPIE, Las Vegas (2016a)

Bates, S.R.G., Farrow, I.R., Trask, R.S.: 3D printed polyurethane honeycombs for repeated tailored energy absorption. Mater. Des. 112, 172-183 (2016b)

Cao, S.-T., Li, Z.-S.: An elastoplastic damage constitutive model for confined concrete under uniaxial load. Eng. Mech. 34(11), 116-125 (2017)

Car, E., Oller, S., Onate, E.: An anisotropic elastoplastic constitutive model for large strain analysis of fiber reinforced composite materials. Comput. Methods Appl. Mech. Eng. 185(2-4), 245-277 (2000)

Chen, Q., et al.: Mechanical properties of a hollow-cylindricaljoint honeycomb. Compos. Struct. 109, 68-74 (2014)

Chen, Y., et al.: 3D printed hierarchical honeycombs with shape integrity under large compressive deformations. Mater. Des. 137, 226-234 (2018)

Farahani, R.D., Lebel, L.L., Therriault, D.: Processing parameters investigation for the fabrication of self-supported and freeform polymeric microstructures using ultraviolet-assisted three-dimensional printing. J. Micromech. Microeng. 24(5), 055020 (2014)

Forquin, P., et al.: Experimental Approach and Modeling of the Compressive Behaviour of Two SiC Grades. J. Phys. IV JP 10(9), 735-740 (2000)

Galehdari, S.A., Kadkhodayan, M., Hadidi-Moud, S.: Low velocity impact and quasi-static in-plane loading on a graded honeycomb structure; experimental, analytical and numerical study. Aerosp. Sci. Technol. 47, 425-433 (2015)

Gibson, L.J., Ashby, M.F.: Cellular Solids: Structure and Properties, 2nd edn, pp. 1-510. Cambridge University Press, Cambridge (2014)

Hadjistamov, D.: Relationship between the normal stress difference and the shear stress Abhaengigkeit der normalspannungsdifferenz von der schubspannung. Rheol. Hann. 5(1), 29-33 (1995)

Hedayati, R., et al.: Mechanical properties of additively manufactured thick honeycombs. Materials 9(8), 613 (2016)

$\mathrm{Hu}, \mathrm{L}$., You, F., Yu, T.: Effect of cell-wall angle on the in-plane crushing behaviour of hexagonal honeycombs. Mater. Des. 46, 511-523 (2013)

Huang, H., et al.: Theoretical analysis for environment of room with windows covered by honeycomb structure. Acta Energiae Solar. Sin. 18(4), 432-436 (1997)

Huang, H., Shi, M., Ge, X.: Numerical simulation of coupled natural convective and radiative heat transfer in compound honeycomb structure with large diameter. Acta Energiae Solar. Sin. 20(1), 59-63 (1999)

Ippili, R.K., et al.: Modeling and identification of polyurethane foam in uniaxial compression: combined elastic and viscoelastic response. In: 2003 ASME Design Engineering Technical Conferences and Computers and Information in Engineering Conference, September 2, 2003-September 6, 
2003. American Society of Mechanical Engineers, Chicago (2003)

Jin, T., et al.: Size effects on the in-plane mechanical behavior of hexagonal honeycombs. Sci. Eng. Compos. Mater. 23(3), 301-307 (2016)

Jin, X., et al.: Investigation on the static and dynamic behaviors of non-pneumatic tires with honeycomb spokes. Compos. Struct. 187, 27-35 (2018)

Kim, K.-W., et al.: Nondimensional analysis of a two-dimensional shear deformable beam in absolute nodal coordinate formulation. Proc. Inst. Mech. Eng. Part C: J. Mech. Eng. Sci. 232(7), 1236-1246 (2017)

Kobayashi, Y., et al.: Local compression fatigue of peripherally clamped honeycomb sandwich panel. Trans. Jpn. Soc. Mech. Eng. Part A 77(775), 415-426 (2011)

Li, H., et al.: Study on surface asperity flattening in cold quasistatic uniaxial planar compression by crystal plasticity finite element method. Tribol. Lett. 58(3), 46 (2015)

Li, C., et al.: Study of EMR and AE During Coal Fracture Under Quasi-Static Uniaxial Compression Load. J. Environ. Eng. Geophys. 22(4), 385-394 (2017)

Li, Z., et al.: Design-oriented crushing analysis of hexagonal honeycomb core under in-plane compression. Compos. Struct. 187, 429-438 (2018)

Papka, S.D., Kyriakides, S.: In-plane compressive response and crushing of honeycomb. J. Mech. Phys. Solids 42(10), 1499-1532 (1994)

Stansbury, J.W., Idacavage, M.J.: 3D Printing with Polymers: Challenges Among Expanding Options and Opportunities. Elsevier Inc, New York (2016)
Uchiyama, N., Nakashima, N.: Carbon nanotubes/polymer composite honeycomb structure film. Kobunshi Ronbunshu 70(2), 55-62 (2013)

Wang, Z.-G., Yao, S.: An equivalent method to cell magnification of aluminum honeycomb under out-of-plane compression. Explos. Shock Waves 33(3), 269-274 (2013)

Wen, S., Chu, X., Sun, J.: Experimental Investigation of Influence Factors for Compression Strength of Thin Honeycomb Paperboard Box, pp. 581-588. Springer, Berlin (2017)

Yang, B., Ravi-Chandar, K.: Evaluation of elastic T-stress by the stress difference method. Eng. Fract. Mech. 64(5), 589-605 (1999)

Zeng, P., et al.: Long-life and high-areal-capacity lithium-sulfur batteries realized by a honeycomb-like N, P dual-doped carbon modified separator. Chem. Eng. J. 349, 327-337 (2018)

Zhang, L., Li, X.: Facile preparation of honeycomb-structured $\mathrm{TiO}_{2}$ nanofilm via breath figures assembly and coffee ring effect. Mater. Lett. 227, 74-77 (2018)

Zhang, Y., Ruan, D.: A practical saturated sand elastic-plastic dynamic constitutive model and application. In: 2nd International Conference on Structures and Building Materials, ICSBM 2012, March 9, 2012-March 11, 2012. Trans Tech Publications, Hangzhou (2012)

Publisher's Note Springer Nature remains neutral with regard to jurisdictional claims in published maps and institutional affiliations. 